\title{
AFFINITY PROPERTIES OF TRUSS MODEL NODES
}

\author{
Douglas CLYDE \\ Emeritus Professor, The University of Western Australia, Nedlands, Western Australia
}

Keywords: plasticity, lower bound, strut and tie, nodes.

\section{INTRODUCTION}

Strut and tie models consist of axial force members which react on one another and on external supports through nodes, i.e. they are trusses. An analysis of a truss problem is normally entered by reducing it to a line diagram of member centrelines representing the lines along which the stress resultants in the members are intended to act. When forces are concentrated in a line diagram the nodes vanish and become points thus ensuring that member forces are concurrent and that moment equilibrium is satisfied at the nodes and this simplifies the problem to one which produces a tractable model.

When fleshing out the results of a truss analysis in order to size members in structural design we use the line diagram to locate the centroidal axes of members, implicitly assuming that it is necessary to maintain the concurrence of axial forces in order to ensure moment equilibrium of nodes. For strut and tie models each node must be detailed so that it is capable of transferring the forces between members. The finite widths of the members generate a common area which is satisfactory as a real node for three force cases but not necessarily for multi-force cases in which case complex nodes have been proposed [1] [2]. We will show that there is a node model in which member forces are not concurrent but moment equilibrium is automatically satisfied in which tithe shape of the boundary is affine to the polygon of forces for the members meeting at the joint. Marti noted to the author [3] that the affinity property presented in this paper is a general statement about properties that he had used in some of his solutions [4], [5].

\section{STRUT AND TIE MODELS}

There has only been a body of theory about strut and tie models in concrete detailing since the seventies, see for example Marti [6]. Nevertheless they were used long before that in an intuitive application of lower theory when permissible stress was supposed to govern design. The author used the method in the design office for the design of steel in pile caps for three piles in the fifties using a text book which dated back to the thirties and possibly before. Similarly, the author was told by Lewis Harding, an intuitive designer in Perth, Western Australia, that he had solved the problem of reinforcing a transfer beam in the early sixties in a very practical way by incorporating spiral reinforcement within the beam with its centroidal axis on the line of the concrete strut needed for a strut and tie solution.

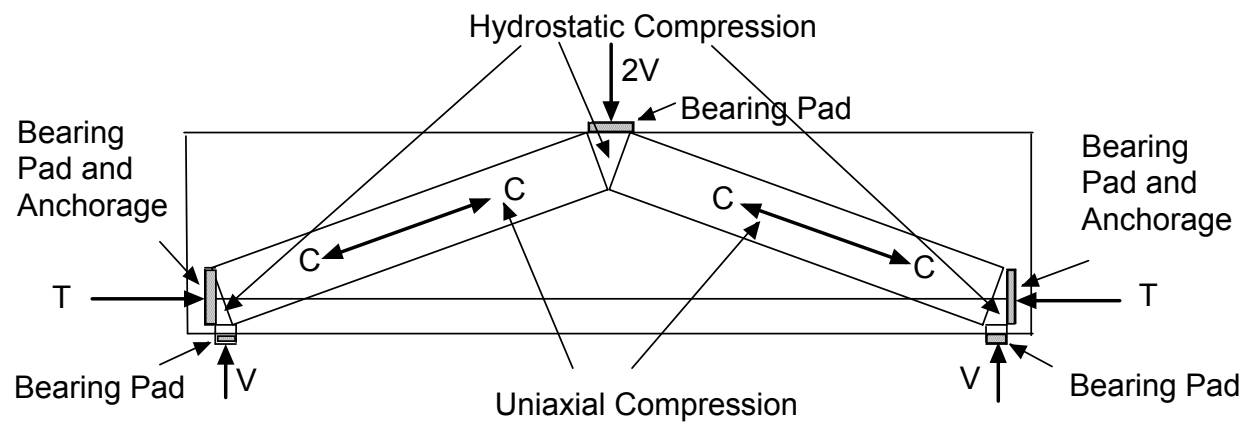

Fig. 1 Classic Strut and Tie Model. 
In the discussion of lower bound approaches to shear in beams the simplest model which is commonly used to introduce the topic is often the strut and tie model of Figure 1.

The nodes are the triangular portions of concrete in hydrostatic compression at the same effective compressive failure stress as the uniaxial axial stress in the struts, $v \mathrm{f}^{\prime}{ }_{c}$. Figure 2 shows the left hand support node which is a triangle acted on by forces $\mathrm{C}, \mathrm{V}$ and $\mathrm{T}$, which are in equilibrium. The stress resultants act through the mid points of the sides and are normal to the sides, so that by Euclidean geometry they intersect in a point and moment equilibrium is automatically satisfied. The forces must form a closed triangular force polygon in order to satisfy horizontal and vertical equilibrium. Because the sides of the node are proportional to the forces and are normal to them the node becomes a linear transformation of the force triangle rotated through $90^{\circ}$, in the sense opposite to the sense with which the vectors are selected going around the outside of the node when constructing the force polygon.

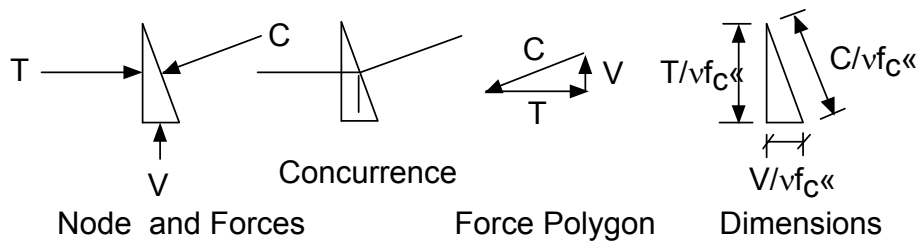

Fig. 2 Node Equilibrium.

These properties of triangular nodes are grasped intuitively so that the construction of strut and tie systems is readily undertaken by hand [5] and may also be automatically generated by computer programme [1]. The location of the reaction nodes is controlled by the location of the force T defined by the centroid of the tension reinforcement and the location of the external reaction, $\mathrm{V}$, and Marti notes in [5] "In developing the truss geometry, the designer must take account the necessary dimensions of the concrete struts and possible placing and detailing of the reinforcement. An iterative procedure is normally required. Starting from an assumed initial truss the necessary strut widths can be determined, then the truss geometry can be modified and the procedure repeated until a satisfactory solution is obtained."

\section{MULTI FORCE NODES}

When there are more than three forces at a node there is no longer a unique relationship between them. As is well known from the solution of statically determinate trusses by equilibrium of the nodes, a node cannot be solved by statics until all but two of the bar forces have been derived by solving for global equilibrium and solving other joints to derive sufficient known forces. In such a case the node for a strut and tie solution becomes more complex. In order to address this Schlaich [1] [2] worked with the requirements for a node which he found in Marti's paper [5] on truss models in detailing from which he chose four out of the five concurrent forces [3].

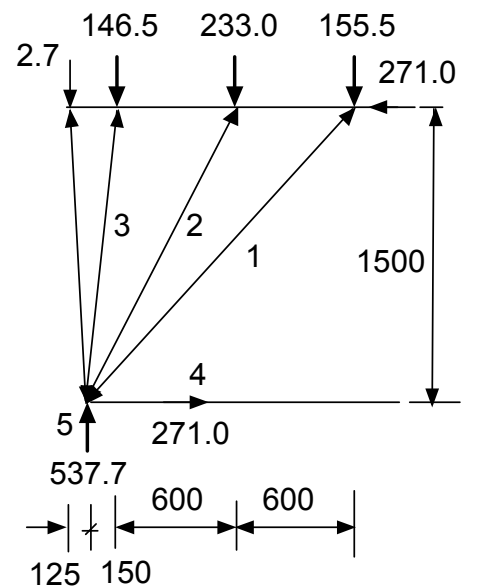

Fig. 3 Marti's Beam Reaction Detail. 
Schlaich simplified the problem by omitting "the lightly loaded strut inclined to the left" [3] and took a direct approach to constructing the node detail, starting with the observation that, unlike the case of the triangular node, the intersection of the implicit concrete strut edges based on the statics of Marti's design define an area which cannot be chosen as the node because the stresses on the boundary would not be in equilibrium with the zero transverse stress in the concrete struts.

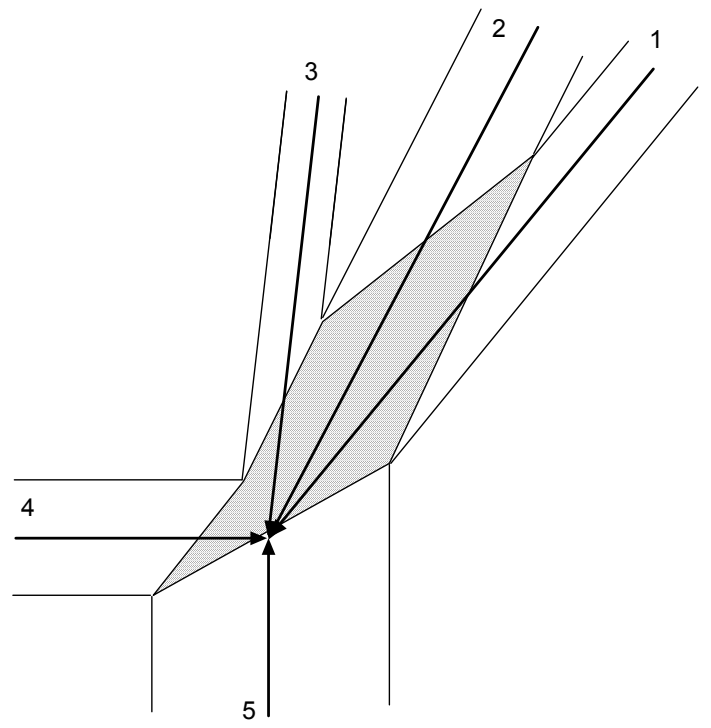

Fig. 4 Schlaich's Starting Point.

In order to satisfy equilibrium at boundaries he then developed a method of systematically combining pairs of strut forces into a new short strut and combining that in turn with a third strut until all struts are accommodated (Figure 5a). He also noted that the forces satisfy a force polygon (Figure $5 b)$.
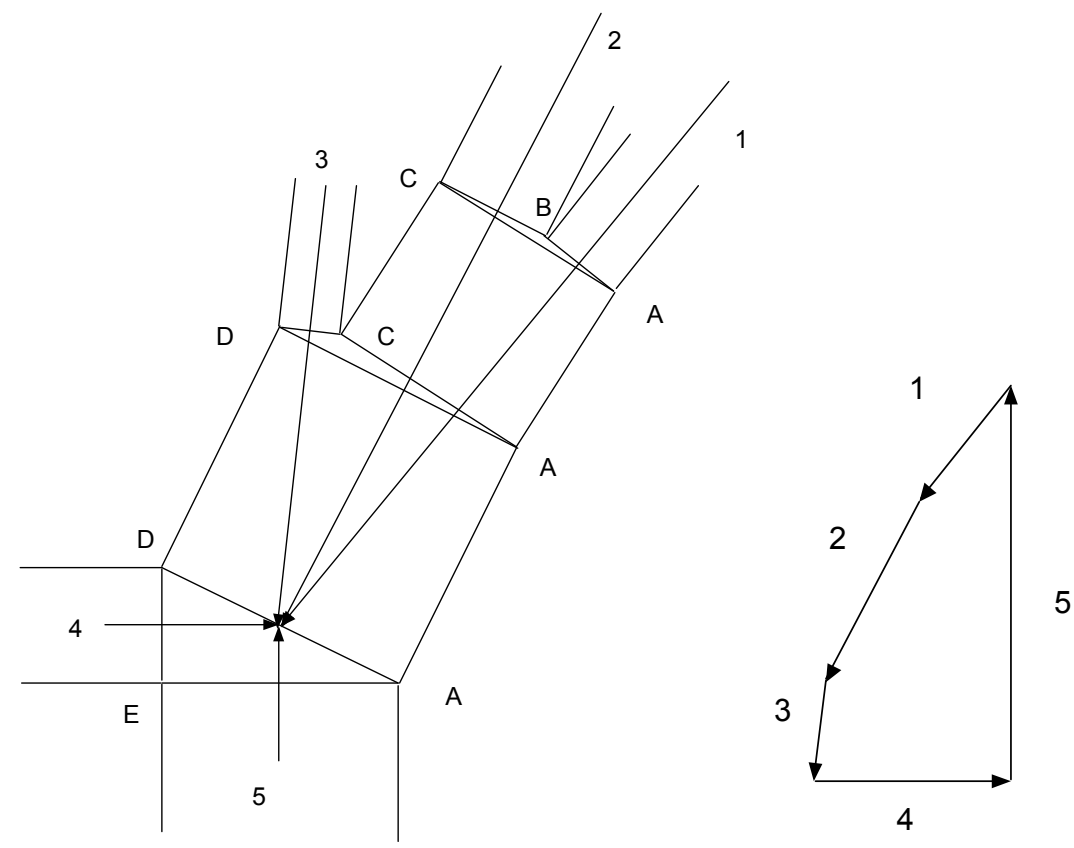

Fig. 5 a. Complex Node. b. Force Polygon.

The force polygon in Figure 5 is once again composed of lines which are proportional to the widths of the individual concrete struts selected in anti-clockwise sequence. In Figure 6a a node is shown in which the length of the force vectors are the same as in the force polygon in Figure 5 and the faces of 
the node normal to them are drawn as the same length as the vectors. This produces a node shape which is again the force polygon rotated clockwise through $90^{\circ}$.
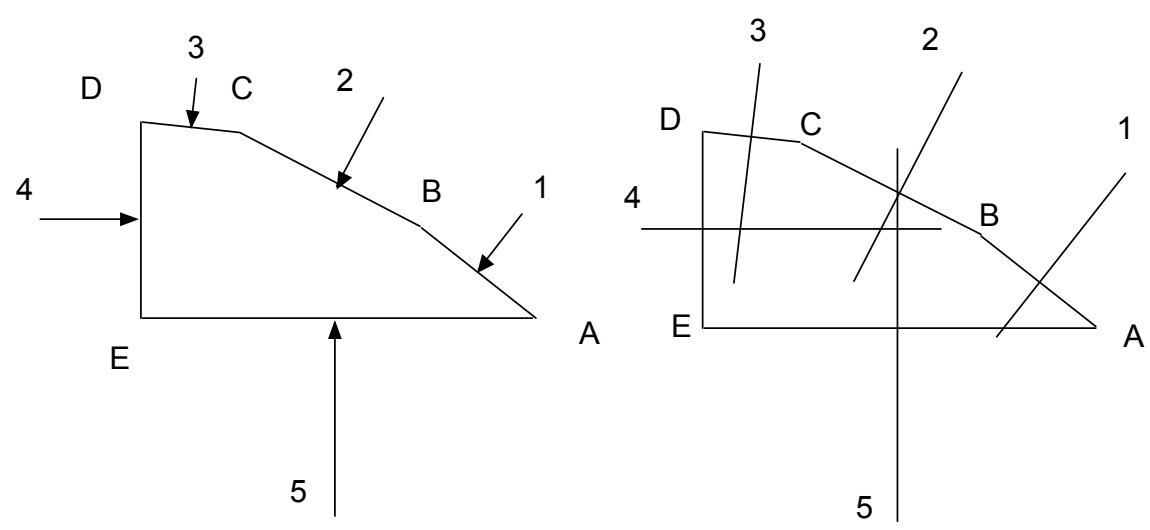

Fig. 6. a. Strut Actions on Node. b. Non-concurrence of Actions

If the strut forces are all carried at a stress of $\sigma_{\mathrm{c}}$ they will be in equilibrium with a hydrostatic stress of $\sigma_{\mathrm{c}}$ in node. It is axiomatic that the hydrostatic system is in moment equilibrium. Analytically we can confirm this. If we join $A$ to $C$ and $A$ to $D$ we have reconstituted sub-nodes $A B C, A C D$ and $A D E$ in Schlaich's complex node. Face $A C$ in $A B C$ is in equilibrium, including moment equilibrium, with face $A C$ in ACD but the strut separating them has vanished. Similarly there is moment equilibrium between $A C D$ and $A D E$. Hence we can always show that moment equilibrium of the vectors in a node which is geometrically similar to a force polygon is satisfied by breaking it down to triangular sub-nodes.

It is not initially obvious how to use a force polygon in a design if we start with Schlaich's depiction of forces 4 and 5 as transmitted by concrete struts. It could, for instance, be that the node is an internal one in a deep beam with an opening such as Schlaich examined in his doctoral dissertation [1] in which case it would be necessary to make an arbitrary decisions about the location of one point on the polygonal boundary of the node and go through several iterations of refining the member geometry in order to freeze the design. However, if the node which he examines is cast in the context from which his data was taken, that of a reaction point in a beam, several parameters in the analysis are automatically set. We will examine this in the following section.

\section{MARTI'S BEAM REACTION}

Recasting the nodal problem in the context of a beam reaction makes it more tractable (Figure 7). It has been noted by Marti that, for a given concrete strength, the positions of $A, E$ and $D$ are invariant under choice of struts with which to transfer the vertical load on the top of a beam to the support. This is because force 4 is determined by the force in the reinforcement which must be transmitted to the back of the node and force 5 is the equilibrant of the vertical loads. The horizontal components of the interface of the other struts with the node are proportional to the vertical components of the strut forces. Hence strut 1 has a vertical component of $155.5 \mathrm{kN}$ and the horizontal component of its interface with the node is $155.5 / \mathrm{vf}^{\prime}{ }_{\mathrm{c}}$ as seen in Figure $7 \mathrm{~b}$. Similarly for struts 2 and 3 the projected width of the struts depends on the vertical component of the force in the strut. It is a straightforward geometrical problem to use this to construct the face, starting either from $A$ or from $D$.

Although the geometry of the node at the reaction and of the struts can be uniquely defined in this way for Marti's beam in Figure 7 this is because the top chord is treated as a stringer. In the general case the top chord is a concrete strut. This not only requires the design of nodes in the top chord to be examined, which will be in the next section, but also leads to the need for iterative procedures in order to arrive at a final solution. 


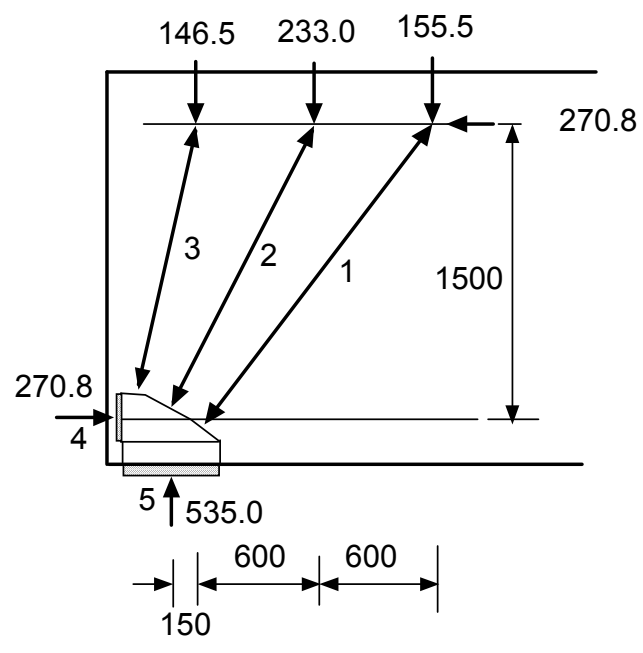

Fig. 7 (a) Force polygon as node.

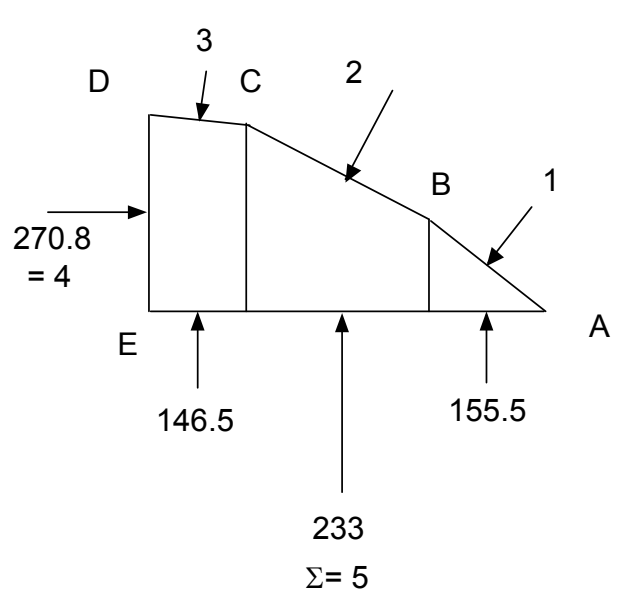

(b) Vertical equilibrium of node.

Another example by Marti in references [4] and [6] is given in Figure 8. It is the case of a uniformly distributed load and again has a node at the support which does not rely on concurrence. There are not discrete forces acting on its boundary but normal stresses. Marti's diagram showed the stresses acting normally on the boundary and he gave a full mathematical solution for both the support node and the top chord compressive zone. He proved that both have parabolic boundaries and in [4] he included the envelope curve for the normals to parabola AC, and the full stress systems in the fans and in DEF.

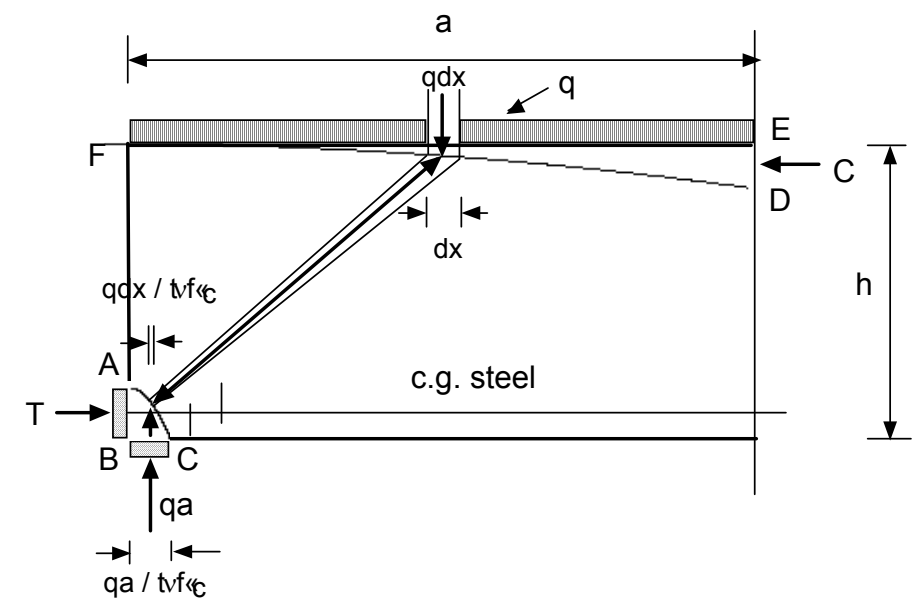

Fig. 8. Marti's Uniformly Loaded Beam Case.

We will use a simpler analysis in the form of a direct statics approach similar to the one proposed above for point loads in order to evaluate the nodal boundary. The vertical projection of the struts will be constant at $(\mathrm{h}-\omega) \quad \omega$ is the symbol Marti uses for the flexural stress block of height DE (= $A B)$ at mid-span. The slope of the strut is therefore given by:

$$
\frac{d y}{d x}=\frac{(x+d x / 2)-1 / n(x+d x / 2)}{(d-\omega)} \text { where } n=v f_{c}^{\prime} / q
$$

Hence the face of the bottom, but not the top, stress block is normal to this so that: 


$$
\frac{d y}{d x}=-\frac{n-1}{n} \frac{(x+d x / 2)}{(d-\omega)}
$$

If $d x$ is allowed to tend to zero this will generate the inverted parabolas with a negative slope proportional to $\mathrm{x}$ which are Marti's solutions for the reaction node and the flexural compression zone.

\section{TOP CHORD NODES IN BEAMS}

Although Schlaich proposes the concurrent treatment for top chord nodes in a beam (figure 9a) it is easy to convert the complex node to a simple force polygon transformation (Figure $9 \mathrm{~b}$ ) and to demonstrate that there is no resultant moment on the node.
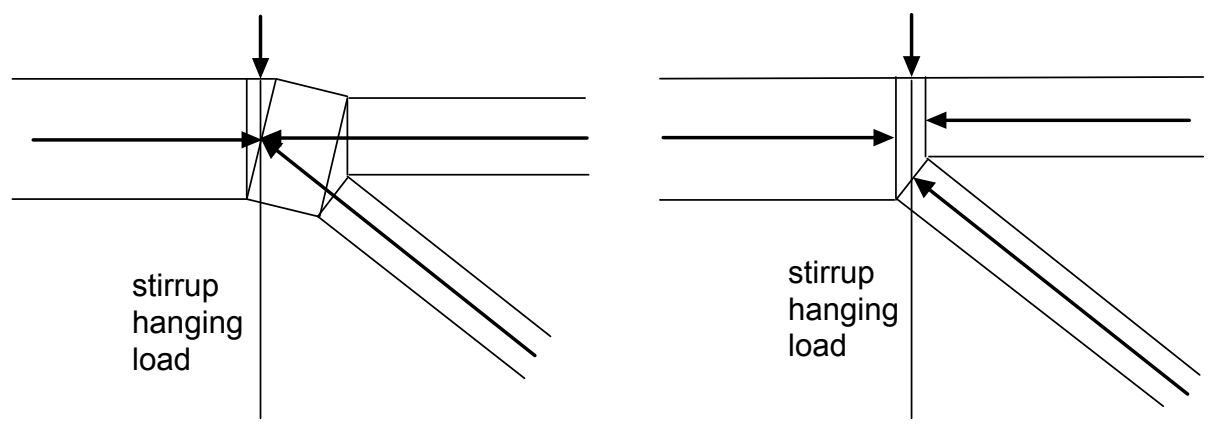

Fig. 9 a. Node proposed by Schlaich. b. Node based on force polygon.

This version has the advantage of locating the top boundary of the compression force in a straight line which in a real beam would represent the top surface of the beam. It does not require the hanging force to be directly applied to the surface, because it is only required to be fully developed at the level where the boundary of the inclined strut meets the smaller axial compressive force so that it is feasible for a stirrup with cover to generate the force provided checking of the steel detailing against the strut dimensions is carried out by the designer.

There is now a degree of freedom to the geometry at both ends of the inclined strut which would not be there if the forces were concurrent so that harmonisation of the geometry at the ends would require some sort of iterative computation.

\section{DISCUSSION}

These solutions show the importance of providing a depth of competent concrete against which inclined struts can react. The author suggests that we have not paid sufficient attention to the general implications arising from this in connection with shear failure of beams. In particular if an inclined flexure shear crack intersects the flexure steel away from the support it is possible that the integrity of the beam would be enhanced by ensuring that the flexure steel is distributed over an area appropriate to the strut force. This is a variable which is not considered when undertaking experiments on size effect and we have tended to think that the competence of the bottom flange is solely influenced by cover.

For example, in Japanese tests with beams varying from $100 \mathrm{~mm}$ to $3 \mathrm{~m}$ effective depth [7] the bars in the three deepest beams are distributed vertically over depths of the same order $(100 \mathrm{~mm}$ to $120 \mathrm{~mm}$ ) even though all other dimensions are kept to scale. If the depth for the $3 \mathrm{~m}$ beam were three times that of the $1 \mathrm{~m}$ beam it would be $300 \mathrm{~mm}$ instead of $120 \mathrm{~mm}$. With bars well distributed over this depth there would be potential for a more competent transfer of force. Furthermore the cover of the $100 \mathrm{~mm}$ beam is $20 \mathrm{~mm}$ and it only increases to $80 \mathrm{~mm}$ for the $3 \mathrm{~m}$ beam thus further reducing the relative distance of the centroid of the steel from the surface.

Nevertheless attention to providing well distributed flexural steel over an area of concrete which is capable of resisting a compression force equal and opposite to the yield force of the steel will not account completely for the size effect and this is only proposed as a factor affecting the size effect which has been overlooked among the others such as concrete strain and crack distribution. 


\section{CONCLUSIONS}

It has been shown that:

- It is not necessary for the forces in multi-force nodes in strut and tie methods of beam design to be concurrent, if the stress state in the node is uniform hydrostatic compression, but that it is sufficient for the forces to be applied across and normal to plane faces of the node through an axial stress which is equal to the hydrostatic stress within the node, and

- The shape of the node will be affine to the force polygon for the forces acting on the node, and corresponds to a rotation of the force polygon through $90^{\circ}$ in the opposite sense to that of the sequence in which the forces are chosen.

The models presented provide a focus on how the forces are transferred between the steel and the concrete which could provide the basis for greater control of variables in future experiments on size effects.

\section{REFERENCES}

[1] Schlaich, M., Computerunterstützte Bemessung von Stahlbetonscheiben mit Fachwerk Modellen, Bericht Nr. 1, Professur für Informatik ETH Zürich, 1989, 127 pp.

[2] Schlaich, M. and Anagnostou, G., "Stress Fields for Nodes of Strut-and-Tie Models", ASCE Journal Structural Engineering, 116, 1, 1990, pp 13-23.

[3] Marti, P., Private Communication with Author. Facsimile dated $6^{\text {th }}$ January 1992 PM/Nö.

[4] Marti, P., Zur Plastischen Berechnung von Stahlbeton Bericht Nr. 104, Institut für Baustatik und Construktion ETH Zürich, 1980, 176 pp.

[5] Marti, P., "Truss Models in Detailing", ACl Concrete International, 7(12), 1985, pp 66-73.

[6] Marti, P., "Basic Tools of Reinforced Concrete Beam Design", ACl Journal, 82(1), 1985, pp 46 -56 .

[7] Iguro, H, Shioya, T, Nojiri, Y. and Akiyama. H., "Experimental Studies on Shear Strength of Large Reinforced Concrete Beams under Uniformly Distributed Load." Concrete Library of JSCE, 5, 1985, pp137-154. 
Morley Symposium on Concrete Plasticity and its Application. University of Cambridge $23^{\text {rd July, } 2007}$ 